\title{
SURGICAL PROCEDURES FOR NEUROENDOCRINE NEOPLASMS OF THE APPENDIX - A CONSENSUS GUIDELINE
}

\author{
Paulina Vladova and Sergey Iliev \\ Department of Coloproctological and Purulent-Septic Surgery, \\ University Hospital of Pleven, Medical University of Pleven, Bulgaria
}

\begin{abstract}
INTRODUCTION: According to the consensus and the recommendations of the European Neuroendocrine Tumor Society (ENETS), the annual incidence rate of neuroendocrine appendicular tumours is $\mathbf{0 . 1 5 -}$ $0.6 / 100000$. They are little more common in women aged between 40 and 50 years. These neoplasms amount to $30-80 \%$ of all appendicular tumours. Their evolution is, usually, asymptomatic and they are diagnosed accidentally during conventional or laparoscopic appendectomy. In the past, the surgical approach used to depend on tumour localization. At presence, this localization is not a decisive factor in the choice of a surgical approach. Our purpose was to demonstrate the consensus guideline and the recommendations of ENETS 2017 for surgical procedures in neuroendocrine appendicular neoplasms and to analyze the difference in the survival rate following various surgical interventions.
\end{abstract}

MATERIAL AND METHODS: Literature overview included studies dealing with the surgical treatment of the neuroendocrine appendicular neoplasms including the most recent consensus guideline of ENETS 2017 on the topic.

RESULTS: Our analysis revealed that according to ENETS 2017, tumour size, localization and tumour cell invasion into the mesoappendix determined the type and volume of surgical intervention. For tumours less than $1 \mathrm{~cm}$ (T1 according to ENETS pathological classification), a simple appendectomy was recommended. In $\mathrm{T} 1$ tumours, this surgical procedure resulted in $100 \%$ survival rate.

CONCLUSION: ENETS 2017 recommends to observe the last established consensus guideline despite the fact that the studies up-to-now do not show any significant difference in the survival rate after appendectomy because of a neuroendocrine tumour of the appendix and right hemicolectomy. Scr Sci Med 2017;49(3):11-14

Keywords: neuroendocrine appendicular neoplasms, ENETS consensus guideline, appendectomy, right hemicolectomy

\footnotetext{
Address for correspondence:

Paulina Vladova

Department of Coloproctological and Purulent-Septic

Surgery

University Hospital of Pleven

Medical University of Pleven, Bulgaria

1 Kliment Ohridski St, 5803 Pleven

e-mail:p.t.vladova@abv.bg
}

Received: July 21, 2017

Accepted: September 15, 2017

\section{INTRODUCTION}

The annual incidence rate of neuroendocrine appendicular tumours is $0.15-0.6 / 100000$ (1). They represent 30 to $80 \%$ of all appendicular neoplasms (1). They are little more common in women aged between 40 and 50 years. They are also observed in children aged between 4.5 and 19.5 years, however, there is no standardized population-based data for this age group yet. The prognosis of these neoplasms is good. In a series of studies, five-year survival rate 
is $100 \%$ or close to $100 \%$ in highly-differentiated tumours $(2,3)$. In large cohort studies involving tumors of varying degrees of differentiation, this rate is between $70 \%$ and $85 \%$ (4). However, it is much lower in patients at advanced stage of the disease and presenting with distant metastases. In such cases, it ranges from $12 \%$ to $28 \%$.

Seventy percent of neuroendocrine appendicular tumours are located at the tip of the appendix (1). Their evolution is, usually, asymptomatic and they are diagnosed accidentally during conventional or laparoscopic appendectomy (5). They persist either asymptomatically, or in the presence of metastasis. Besides they may exhibit symptoms associated with the localization of the metastases. Carcinoid syndrome is rarely observed with these neoplasms (6). There are no imaging studies characterized by high informative and specific probative value.

Somatostatin receptor imaging and positron emission tomography can be used to prove distant metastases. Chromogranin A is used as a laboratory tumour marker for an advanced disease, however, it is not validated for diagnosis and follow-up of these neoplasms yet.

According to consensus decisions and recommendations from the European Neuroendocrine Tumor Society (ENETS) 2011 and 2017, the size, localization and the tumor cell invasion into the mesoappendix determine the surgical therapy. Two surgical techniques are applicable to these tumours: simple appendectomy and oncological right hemicolectomy (1). The most recent recommendations and consensus guideline of March 2017 set out precise selection criteria for specific surgery which is the most appropriate one for these patients.

Our purpose is to present the consensus guideline and the recommendations of ENETS 2017 for surgical procedures in neuroendocrine appendicular neoplasms and to analyze the difference in the survival rate following various surgical interventions.

\section{MATERIAL AND METHODS}

Literature overview includes studies about surgical treatment of neuroendocrine neoplasms of the appendix including the last consensus guideline of ENETS 2017 on the topic.

\section{RESULTS}

Our analysis reveals that according to ENETS 2017, tumour size, localization and tumour cell invasion into the mesoappendix determine the type and volume of surgical intervention. For tumours less than $1 \mathrm{~cm}$ (T1 according to ENETS pathological classification), a simple appendectomy is recommended. In $\mathrm{T} 1$ tumours, this surgical procedure leads to $100 \%$ survival rate.

\section{DISCUSSION}

Tumours sized between 1 and $2 \mathrm{~cm}$ (T2) prove to be a challenge for surgeons because of the risk of metastasis and/or relapse. Most often, metastases occur in tumours larger than $1.5 \mathrm{~cm}$ (7). Tumours sized above $2 \mathrm{~cm}$ (T3) are rare, less than $10 \%$ of all the neuroendocrine appendicular neoplasms. The risk of metastasis in T2 tumours reaches up to $40 \%(8,9)$. The European Neuroendocrine Tumors Society recommends oncological right hemiclectomy in T3 tumors. The tumours emerging from the appendix and infiltrating adjacent structures (T4) require systemic oncological evaluation including initial right hemicolectomy.

Sixty to $75 \%$ of the neuroendocrine appendicular neoplasms are located at the tip, 5 to $20 \%$ of them are placed in the middle, and only $10 \%$ of them are at the base of the appendix. There is no strict correlation between survival rate and tumour localization, however, it is assumed that the tumours at the appendicular base are more prone to metastasis $(10,11)$.

An invasion into the mesoappendix is observed in up to $20 \%$ of adults and in up to $40 \%$ in children $(12,13)$. An invasion of more than $3 \mathrm{~mm}$ results in a more aggressive course of the disease.

The oncological right hemicolectomy is recommended for tumours sized above $2 \mathrm{~cm}$. In T1 tumours, the simple appendectomy is sufficient. However, this intervention is not sufficient in cases either when the tumour is located at the appendicular base, or when it infiltrates the mesoepithelium at a depth of more than $3 \mathrm{~mm}$. In T2 tumours, lymph node locations as well as distant metastases are likely. Therefore, the simple appendectomy in these tumours is not always radical and the recurrence is common, especially in young subjects. The risk of relapse increases in tumours sized above $1.5 \mathrm{~cm}$. 
ENETS recommends oncological right hemicolectomy if one of the following criteria exists:

$\diamond$ moderately differentiated tumour (G2)

$\diamond$ vascular invasion (V1)

$\diamond$ lymphatic invasion (L1), and

$\diamond$ infiltration into the mesoappendix deeper than $3 \mathrm{~mm}$.

In T3 tumours, oncological right hemicolectomy is performed either as an initial surgical intervention, or as a second intervention after initial appendectomy. The neuroendocrine appendicular carcinomas (G3) are treated as adenocarcinomas.

ENETS 2017 assumes that in tumours less than $2 \mathrm{~cm}$, the appendectomy is sufficient, regardless of tumour location. Right hemicolectomy is justified only in cases of tumours sized 1 to $2 \mathrm{~cm}$ with positive or unclear resection margins, invasion into the mesoappendix, high proliferative index, or vascular invasion. Tumours sized more than $2 \mathrm{~cm}$ should be treated by oncological right hemicolectomy.

\section{CONCLUSION}

Well-sized neuroendocrine appendicular tumours smaller than $1 \mathrm{~cm}$ do not require any followup. For tumours sized between 1 and $2 \mathrm{~cm}$, followup is desirable, but not recommended. For tumours sized over $2 \mathrm{~cm}$, mandatory follow-up at 6 and 12 months and then once yearly is recommended, although there is no strictly validated standard yet.

The neuroendocrine appendicular tumour is based on specific pathological and clinical criteria developed and recommended by the European Neuroendocrine Tumor Society. Despite currently available studies demonstrating the lack of significant difference in survival rates after appendetomy compared to right hemicolectomy, ENETS 2017 recommends to strictly observe the most recent established consensus.

\section{REFERENCES}

1. Pape UF, Niederle B, Costa F, Gross D, Kelestimur F, Kianmanesh R, et al.; Vienna Consensus Conference participants. ENETS Consensus Guidelines for Neuroendocrine Neoplasms of the Appendix (Excluding Goblet Cell Carcinomas). Neuroendocrinology. 2016;103(2):144-52. doi: $10.1159 / 000443165$.
2. Hsu C, Rashid A, Xing Y, Chiang YJ, Chagpar $\mathrm{RB}$, Fournier KF, et al. Varying malignant potential of appendiceal neuroendocrine tumors: importance of histologic subtype. J Surg Oncol. 2013;107(2):136-43. doi: 10.1002/jso.23205.

3. Garcia-Carbonero R, Capdevila J, Crespo-Herrero G, Díaz-Pérez JA, Martínez Del Prado MP, Alonso Orduña $V$, et al. Incidence, patterns of care and prognostic factors for outcome of gastroenteropancreatic neuroendocrine tumors (GEP-NETs): results from the National Cancer Registry of Spain (RGETNE). Ann Oncol. 2010;21(9):1794-803. doi: 10.1093/annonc/mdq022.

4. Quaedvlieg PF, Visser O, Lamers CB, Janssen-Heijen ML, Taal BG. Epidemiology and survival in patients with carcinoid disease in The Netherlands. An epidemiological study with 2391 patients. Ann Oncol. 2001;12(9):1295-300.

5. Boxberger N, Redlich A, Böger C, Leuschner I, von Schweinitz D, Dralle H, et al. Neuroendocrine tumors of the appendix in children and adolescents. Pediatr Blood Cancer. 2013;60(1):65-70. doi: $10.1002 / \mathrm{pbc} .24267$.

6. Goede AC, Caplin ME, Winslet MC. Carcinoid tumour of the appendix. Br J Surg. 2003;90(11):131722. doi: 10.1002/bjs. 4375 .

7. Volante M, Daniele L, Asioli S, Cassoni P, Comino A, Coverlizza $S$, et al. Tumor staging but not grading is associated with adverse clinical outcome in neuroendocrine tumors of the appendix: a retrospective clinical pathologic analysis of 138 cases. Am J Surg Pathol. 2013;37(4):606-12. doi: 10.1097/ PAS.0b013e318275d1d7.

8. Moertel CG, Weiland LH, Nagorney DM, Dockerty MB. Carcinoid tumor of the appendix: treatment and prognosis. N Engl J Med. 1987 Dec 31;317(27):1699-701. doi:: 10.1056/ NEJM198712313172704.

9. Fornaro R, Frascio M, Sticchi C, De Salvo L, Stabilini C, Mandolfino F, et al. Appendectomy or right hemicolectomy in the treatment of appendiceal carcinoid tumors? Tumori. 2007;93(6):587-90.

10. Pape UF, Perren A, Niederle B, Gross D, Gress T, Costa F, et al.; Barcelona Consensus Conference participants. ENETS Consensus Guidelines for the management of patients with neuroendocrine neoplasms from the jejuno-ileum and the appendix including goblet cell carcinomas. Neuroendocrinology. 2012;95(2):135-56. doi: 10.1159/000335629. 
11. Tang LH. Epithelial neoplasms of the appendix. Arch Pathol Lab Med. 2010;134(11):1612-20. doi: 10.1043/2010-0073-CCR.1.

12. Bamboat ZM, Berger DL. Is right hemicolectomy for 2.0-cm appendiceal carcinoids justified? Arch Surg. 2006;141(4):349-52; discussion 352. doi:10.1001/archsurg.141.4.349.

13. Dunn JP. Carcinoid tumours of the appendix: 21 cases, with a review of the literature. $\mathrm{N} Z \mathrm{Med}$. 1982;95(701):73-6. 